\title{
On the Motion of the Large-Scale Baroclinic Disturbance Including the Non-Geostrophic Wind Component
}

\author{
by \\ M. Aihara \\ Meteorological Research Institute, Tokyo \\ (Received January 10, 1961)
}

\begin{abstract}
Equations of motion, in a primitive form, of adiabatic change and continuity relation are used to examine the stability properties of the large-scale baroclinic disturbance including the non-geostrophic effect.

Especially, in the longer and shorter wavelengths, there seems to be no appreciable change in the stability criterion obtained so far.

The fundamental equations involve three kinds of propagating waves. Two of them are expressed by combination of the Rossby wave and the inertial internal gravity wave, and the other is the socalled meteorologically significant wave, which propagates westward with a very small velocity.

By using data carefully analysed, the following quantity is estimated ;

$$
R \equiv K_{x t} / K_{\varphi l},
$$

in order to know the ratio of time rates of the kinetic energy of the divergent and rotational component of the wind.

Simultaneously, $G$-number is estimated for 300,500 and $700 \mathrm{mb}$ levels of the atmosphere.
\end{abstract}

\section{Fundamental equations}

The fundamental equations taken up are the equations of motion of adiabatic change, continuity relation and the hydrostatic assumptions. They take the following forms:

$$
\left[\begin{array}{rl}
\frac{\partial \boldsymbol{V}}{\partial t} & =-\boldsymbol{V} \cdot \nabla \boldsymbol{V}-\omega \frac{\partial \boldsymbol{V}}{\partial p}-f \boldsymbol{k} \times \boldsymbol{v}-\nabla \phi \\
\frac{\partial}{\partial t} \frac{\partial \phi}{\partial p} & =-\boldsymbol{V} \cdot \nabla \frac{\partial \phi}{\partial p}+\frac{\alpha}{\theta} \frac{\partial \theta}{\partial p} \omega \\
\nabla \cdot \boldsymbol{V} & =-\partial \omega / \partial p \\
\frac{\partial \phi}{\partial p} & =-\alpha,
\end{array}\right.
$$

Here, $(x, y, p, t)$ coordinate system is adopted, $x$ and $y$ directed positively eastward and northward respectively, and $p$ is taken positively downward. $V, \nabla$ stand for 
the two-dimensional wind vector of components $(u, v)$ and the vector operator, respectively, $f$ the Coriolis parameter, $\phi$ the geopotential height, $\omega$ the vertical $p-$ velocity, $\alpha$ the specific volume, $\theta$ the potential temperature and $\boldsymbol{k}$ the vertical unit vector. If we transform the equation of motion by applying the rotation and divergence operation, then we have the vorticity and divergence equation in the following way:

$$
\left[\begin{array}{l}
\frac{d}{d t}(\zeta+f)=-(\zeta+f) \cdot \delta-\boldsymbol{k} \cdot \nabla \omega \times \frac{\partial \boldsymbol{V}}{\partial p} \\
\frac{d}{d t} \delta=-\delta^{2}+2 \boldsymbol{K} \nabla u \times \nabla v-\nabla \omega \frac{\partial \boldsymbol{V}}{\partial p}+\boldsymbol{k} \nabla f \times \boldsymbol{V}+f \zeta-\nabla^{2} \phi,
\end{array}\right.
$$

where, $\zeta$ denotes the vertical component of the relative vorticity, $\delta$ the horizontal divergence, and $\frac{d}{d t} \equiv \frac{\partial}{\partial t}+V \cdot \nabla+\omega \frac{\partial}{\partial p}$, the individual operator.

For the sake of simplicity, we will consider the simple two-level baroclinic

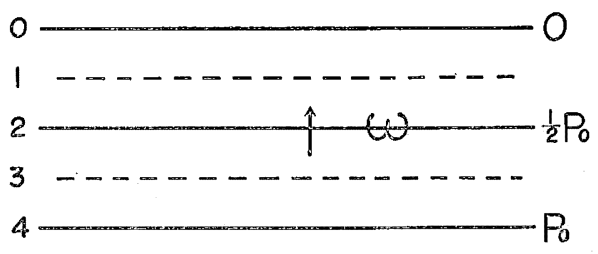

Fig. 1. Two-layer representation of the atmosphere. The boundary condition $\omega=0$ at $p=0, p_{0}$. atmosphere. The atmosphere is divided into two layers of the levels 1 and 3 and the interface is named the level 2. The boundary conditions at the top and the bottom of the atmosphere are $\omega=0$, for we simply consider the plane earth. On the other hand, the vertical velocities at the levels 1 and 3 are necessary in order to evaluate the second term on the right hand side of the vorticity equation, and

the third term on the right hand side of the divergence equation. They are assumed to be expressed by a simple average of quantities at the upper or lower and middle layers, as used by ELIASSEN, that is to say,

$$
\left\{\begin{array}{l}
\omega_{1}=\frac{\omega+\omega_{0}}{2}=\frac{1}{2} \omega \\
\omega_{3}=\frac{\omega+\omega_{10}}{2}=\frac{1}{2} \omega
\end{array}\right.
$$

where $\omega$ means the vertical velocity at the mid-level 2 of the simple baroclinic atmosphere.

\section{Simple considerations on stability properties of the baroclinic atmosphere includ- ing the non-geostrophic effect}

In this section, we shall concern ourselves with the effect of the existence of the non-geostrophic wind component upon the stability properties of the baroclinic atmosphere of the quasi-geostrophic approximation.

The problem will be treated basing upon the linearized perturbation theory. As the fundamental state of the atmosphere, we will assume the following: the presence of a basic current with a constant vertical shear but a horizontally uniform flow, and the wavy type disturbance of an infinite width superposed upon it. The basic current is assumed to satisfy the geostrophic relations, 


$$
\begin{aligned}
& U_{1}=-\frac{1}{f} \frac{\partial \Phi_{1}}{\partial y}, \\
& U_{3}=-\frac{1}{f} \frac{\partial \Phi_{3}}{\partial y} .
\end{aligned}
$$

Here, $U_{1}, U_{3}$ are constant with respect to both time and space. Therefore, the vorticity equations at levels 1 and 3 become

$$
\left[\begin{array}{l}
\left(\frac{\partial}{\partial t}+U_{1} \frac{\partial}{\partial x}\right) \cdot \zeta_{1}+\beta \cdot v_{1}=-f_{0} \cdot \delta_{1} \\
\left(\frac{\partial}{\partial t}+U_{3} \frac{\partial}{\partial x}\right) \cdot \zeta_{3}+\beta \cdot v_{3}=-f_{0} \cdot \delta_{2},
\end{array}\right.
$$

where $\beta$ denotes the Rossby factor, $f_{0}$ the Coriolis parameter at a certain representative latitude. The corresponding divergence equations are written as follows:

$$
\left[\begin{array}{l}
\left(\frac{\partial}{\partial t}+U_{1} \frac{\partial}{\partial x}\right) \cdot \delta_{1}=2 \frac{\Lambda}{p_{0}} \frac{\partial \omega}{\partial x}-\beta u_{1}+f_{0} \zeta_{1}-\frac{\partial^{2} \phi_{1}}{\partial x^{2}} \\
\left(\frac{\partial}{\partial t}+U_{3} \frac{\partial}{\partial x}\right) \cdot \delta_{3}=2 \frac{\Lambda}{p_{0}} \frac{\partial \omega}{\partial x}-\beta u_{3}+f_{0} \zeta_{3}-\frac{\partial^{2} \phi_{3}}{\partial x^{2}} .
\end{array}\right.
$$

The adiabatic equation takes the form

$$
\frac{\partial}{\partial t}\left(\phi_{1}-\phi_{3}\right)=-\bar{U} \frac{\partial}{\partial x}\left(\phi_{1}-\phi_{3}\right)+f_{0} \Lambda\left(v_{1}+v_{3}\right)+\frac{p_{0}}{2 \sigma} \omega,
$$

$\sigma$ is a measure of static stability, defined by

$$
\begin{aligned}
& \sigma \equiv\left[\Phi_{p}(\ln \theta)_{p}\right]^{-1}>0, \\
& \omega=-\frac{1}{2} p_{0} \delta_{1}=\frac{1}{2} p_{0} \delta_{3},
\end{aligned}
$$

means the continuity relation at two reference levels. Here, the notations are defined in the following way:

$$
\left[\begin{array}{l}
\bar{U} \equiv \frac{1}{2} \cdot\left(U_{1}+U_{3}\right) \\
\Lambda \equiv \frac{1}{2} \cdot\left(U_{1}-U_{3}\right)
\end{array}\right.
$$

$\bar{U}$ stands here for the mean speed of the current at a middle layer of the atmosphere at level 2 , and $A$ corresponds to the vertical shear of the basic current.

The divergence equation can be rewritten as

$$
\left[\begin{array}{l}
\left(\frac{\partial}{\partial t}+\tilde{U}_{1} \frac{\partial}{\partial x}\right) \cdot \delta_{1}=-\beta u_{1}+f_{0} \zeta_{1}-\frac{\partial^{2} \phi_{1}}{\partial x^{2}} \\
\left(\frac{\partial}{\partial t}+\tilde{U}_{3} \frac{\partial}{\partial x}\right) \cdot \delta_{3}=-\beta u u_{3}+f_{0} \zeta_{3}-\frac{\partial^{2} \phi_{3}}{\partial x^{2}},
\end{array}\right.
$$

where

$$
\left\{\begin{array}{l}
\tilde{U}_{1} \equiv U_{1}+\Lambda \\
\tilde{U}_{3} \equiv U_{3}-\Lambda .
\end{array}\right.
$$


Here, we assume a wave form for the disturbance quantities, that is,

$$
\left(u_{j}, v_{j}, \phi_{j}, \omega\right) \simeq e^{i k(x-c t)}, \quad j=1,3,
$$

and $L=2 \pi / k$, the zonal wavelength of the disturbance. By substitution of the above form into the vorticity, divergence and adiabatic equations by help of the continuity relation, the fourth order equation concerning the propagation speed $C$, the so-called frequency equation is derived namely,

$$
\begin{aligned}
0= & (\bar{U}-C)\left\{\beta-k^{2}\left(U_{1}-C\right)\right\}\left\{\beta-k^{2}\left(U_{3}-C\right)\right\}\left[\beta-k^{2}\left(\tilde{U}_{1}-C\right)+\beta-k^{2}\left(\tilde{U}_{3}-C\right)\right] \\
& -2 f_{0}^{2} k^{2}(\bar{U}-C)\left\{\beta-k^{2}(\bar{U}-C)\right\} \\
& +f_{0}{ }^{2} k^{2} \cdot\left[2 \Lambda^{2} k^{2}+\frac{p_{0}^{2}}{4 \sigma f_{0}^{2}}\left\{\beta-k^{2}\left(U_{1}-C\right)\right\}\left\{\beta-k^{2}\left(U_{3}-C\right)\right\}\right] .
\end{aligned}
$$

This formula is similar to the one already obtained by Thompson (1957), and can be summarized into the form

$$
F(z)=G(z)+N(z)=0
$$

where

$$
\left(\begin{array}{rl}
G(z) & \equiv-C_{I}^{2}\left(1+\varepsilon^{2}\right)\left(z-C_{10}\right)\left(z-C_{20}\right) \\
N(z) & \equiv z\left(z-C_{R}\right)\left(z-C_{R}+\Lambda\right)\left(z-C_{R}-\Lambda\right) \\
z & \equiv \bar{U}-C,
\end{array}\right.
$$

and the notations used have following meanings:

$$
\left[\begin{array}{rl}
\varepsilon^{2} \equiv k^{2} / 8 k_{0}{ }^{2} ; & \text { the non-dimensional parameter relating to the disturb- } \\
& \text { ance wavelength. } \\
C_{R} \equiv \beta / k^{2} & ; \text { Rossby wave speed. } \\
C_{I} \equiv f_{0} / k \quad ; \text { Inertia wave velocity, } & \\
\left(\begin{array}{l}
C_{10} \\
C_{20}
\end{array}\right) \equiv \frac{1}{2}\left[C_{R} \cdot \frac{1+2 \varepsilon^{2}}{1+\varepsilon^{2}} \pm \frac{1}{1+\varepsilon^{2}} \sqrt{C_{R}{ }^{2}+4 \Lambda^{2}\left(\varepsilon^{4}-1\right)}\right] ; & \begin{array}{l}
\text { The wave velocity of the } \\
\text { baroclinic disturbance. }
\end{array}
\end{array}\right.
$$

(i) Especially, if the non-geostrophic component is absent, the frequency equation is reduced to

$$
F(z)=G(z)=0 .
$$

This equation gives the well-known stability criterion obtained by FJORTOFT, of the baroclinic atmosphere for the wave type geostrophic disturbance.

(ii) Another term of the frequency equation

$$
N(z)=0
$$

shows the existence of the four kinds of neutral waves which propagate with the corresponding wave speed without changing the amplitude.

(iii) In the case of the coexistence of both terms, $F(z)=G(z)+N(z)$, the situation becomes more or less complex. Since the frequency equation $F(z)$ is a fourth degree on $Z$, it is rather a complicated task to examine the stability properties of 
the formula $F(z)$ for various ranges of the disturbance wavelength $L$ and the vertical shear of the basic current, $A$. Therefore, we will primarily concern ourselves with the subject of the possibility of the occurrence of new type of instability caused by the presence of the non-geostrophic wind component.

In the baroclinic atmosphere, the existence of a critical wavelength, beyond which the disturbance potential energy is to be transformed into kinetic energy, is proved by EADY and FJ $\phi$ RTOFT, and given by

$$
\frac{1}{8 k_{0}^{2}}=\frac{p_{0}^{2}}{8 \sigma f_{0}^{2}}=\frac{1}{8 f_{0}^{2}} p_{0}^{2}\left[\Phi_{p}(\ln \theta)_{p}\right]=\frac{\mu^{2}}{f_{0}^{2}},
$$

where $\mu$ means the velocity of the internal gravity wave, $f_{0}$ the Coriolis parameter. Here, we will classify the disturbance into a longer and a shorter wave region, according as the wavelength is longer or shorter than the critical value, and examine the behaviours of the disturbance for each wave range.

(I) First, we will concern ourselves with the short wave disturbance.

In this case, the following approximation seems to be plausible in the frequency equation (8):

$$
\varepsilon^{2}>1, \quad \beta=0 \text { or } \quad C_{R}=0 .
$$

Therefore the equation is reduced to a comparatively simple form, but the poly. nomial of the fourth order on $z$.

$$
F(z)=z^{4}-\left(\Lambda^{2}+C_{I}^{2} \varepsilon^{2}\right) \cdot z^{2}-C_{I}^{2} \not^{2} \cdot\left(1-\varepsilon^{2}\right)=0 .
$$

The above equation is immediately solved and gives four kinds of propagation velocities,

$$
z= \pm \frac{1}{\sqrt{2}}\left[\Lambda^{2}+C_{I}{ }^{2} \varepsilon^{2} \pm \sqrt{\left(\Lambda^{2}-C_{I}{ }^{2} \varepsilon^{2}\right)^{2}+4 C_{I}^{2} \Lambda^{2}}\right]^{1 / 2} .
$$

The quantity $C_{I}{ }^{2} \varepsilon^{2}$ in the bracket is independent of the disturbance wavelength $L$,

$$
C_{I}^{2} \varepsilon^{2}=\frac{f_{0}^{2}}{32 \pi^{2}} \cdot L_{0}^{2} \equiv C_{0}^{2} .
$$

Therefore, with the use of the above notation, we have

$$
z= \pm \frac{1}{\sqrt{2}} \cdot\left[\Lambda^{2}+C_{0}^{2} \pm \sqrt{\left(\Lambda^{2}-C_{0}^{2}\right)^{2}+4 C_{I}^{2} \Lambda^{2}}\right]^{1 / 2} .
$$

As immediately seen from the expression, the criterion whether $z$ is a real or a complex number is uniquely decided by the following relation, or the criterion of growth or decay of the disturbance amplitude is given by

$$
\begin{array}{ll}
C_{I} \gtreqless C_{0} & \text { decay } \\
& \text { growth. }
\end{array}
$$

The criterion obtained above is quite similar with the one derived by EADY for the short wave disturbance with the geostrophic approximation. Hence, we may con- 
clude that the stability properties of the simple baroclinic atmosphere does not undergo any noticeable change by the introduction of the non-geostrophic wind component.

(II) For the disturbance with the longer wavelength, the approximation $\varepsilon^{2} \ll 1$, is applicable.

In the long wave range, it may be supposed that the propagation velocity will asymptotically approach to the Rossby wave speed, for the effect of the Rossby factor $\beta$ becomes a predominant factor.

Therefore, we may seek two sets of solution of the frequency equation in the following way :

$$
z=C_{R}+\gamma
$$

where the relation $\left|\gamma / C_{R}\right| \ll 1$ is presupposed.

The frequency equation (8) becomes, with the use of the long wave approximation, $\varepsilon^{2} \ll 1$,

$$
F(z)=\gamma^{4}+C_{R} \gamma^{3}-\left(C_{I}^{2}+\Lambda^{2}\right) \cdot \gamma^{2}-C_{R}\left(C_{I}^{2}+\Lambda^{2}\right) \cdot \gamma-C_{I}^{2} \Lambda^{2}=0 .
$$

By neglecting the higher order terms of $\gamma$, because of the inequality relation $\left|\gamma / C_{R}\right| \ll 1$, (12) is reduced to

$$
\left(C_{I}^{2}+\Lambda^{2}\right) \cdot \gamma^{2}+C_{R} \cdot\left(C_{I}^{2}+\Lambda^{2}\right) \cdot \gamma+C_{I}^{2} \Lambda^{2}=0
$$

Therefore, the quadratic equation of $\gamma$ is easily solved,

$$
\gamma=\frac{1}{2}\left[-C_{R} \pm \sqrt{C_{R}^{2}-4 \frac{C_{I}^{2} \Lambda^{2}}{C_{I}^{2}+\Lambda^{2}}}\right] .
$$

The stability criterion can be derived from the expression within the root sign. Thus we have

$$
\Lambda^{2}=\frac{C_{R}^{2} C_{I}^{2}}{4 C_{I}^{2}-C_{R}^{2}}=\frac{\left(\frac{f_{0}}{2 \pi}\right)^{2} \cdot L^{4}}{\left(\frac{4 \pi f_{0}}{\beta}\right)^{2}-L^{2}} .
$$

The critical wavelength, at which the vertical wind shear of the basic current becomes infinite is approximately given by

$$
L_{C}=\frac{4 \pi f_{0}}{\beta} \simeq 4 \pi a \tan \varphi_{0} \simeq 80,000 \mathrm{~km} \quad \text { at } 45^{\circ} \mathrm{N},
$$

where $\mathrm{a}$ is the radius of the earth, and $\varphi_{0}$ the latitudinal angle. However, the total length of the latitudinal circle at $45^{\circ} \mathrm{N}$ roughly corresponds to $30,000 \mathrm{~km}$, so that the critical wavelength obtained above is rather a fictitious one. The quantities within the root sign of the expression (13) show behaviours quite similar to those of the geostrophic model with respect to the disturbance wavelength $L$. The situation will be clear if we examine the behaviours of the propagation speed $C_{10}$, $C_{20}$ and $\gamma$ for longer wave range; that is to say 


$$
\left\{\begin{array}{l}
\lim _{\varepsilon \rightarrow 0} C_{10,20}=\frac{1}{2}\left[C_{R} \pm \sqrt{C_{R}^{2}-4 \Lambda^{2}}\right] \\
\lim _{\varepsilon \rightarrow 0} \gamma=\frac{1}{2}\left[-C_{R} \pm \sqrt{C_{R}^{2}-4 \Lambda^{2}}\right] .
\end{array}\right.
$$

We can easily understand that the expression within the root sign gives the parabolic relation between the vertical wind shear of the basic current $A$, and the disturbance wavelength $L$, which is one of the characteristics of the baroclinic atmosphere with a constant vertical shear.

The other sets of solution of the frequency equation must be sought for in a different way, since the formula $F(z)=0$ is a ploynomial of fourth degree on variable $z$. In order to get a better approximation to the non-geostrophic wind component of $F(z)$, the following form of solution is assumed:

$$
\begin{aligned}
F(z) & =\left[z^{2}-C_{R} z+\frac{C_{I}^{2} \Lambda^{2}}{C_{I}^{2}+\Lambda^{2}}\right] \cdot W(z) \\
& =\left[z^{2}-C_{R} z+\frac{C_{I}^{2} \Lambda^{2}}{C_{I}^{2}+\Lambda^{2}}\right] \cdot\left(z^{2}+A z+B\right),
\end{aligned}
$$

where the coefficients $A$ and $B$ stand for the undetermined constant, and are to be decided by such a requirement that the best fit for the terms of the second and the third order of $z$ should be obtained instead of the lower-order terms of $z$.

Thus, $A$ and $B$ are determined as follows:

$$
\left\{\begin{array}{l}
B=C_{R}{ }^{2}-C_{I}^{2}-\Lambda^{2}-\frac{C_{I}^{2} \Lambda^{2}}{C_{I}^{2}+\Lambda^{2}} \\
A=-2 C_{R}
\end{array}\right.
$$

so that

$$
W(z)=z^{2}-2 C_{R} \cdot z+\left[C_{R}{ }^{2}-C_{I}{ }^{2}-\Lambda^{2}-\frac{C_{I}^{2} \Lambda^{2}}{C_{I}^{2}+\Lambda^{2}}\right]
$$

This quadratic formula concerning $z$ does not involve the unstable wave. This is easily known from the examination of the discriminant of the formula; for the relation

$$
D \equiv C_{I}^{2}+\Lambda^{2}+\frac{C_{I}^{2} \Lambda^{2}}{C_{I}^{2}+\Lambda^{2}}>0
$$

holds for any wavelength. The corresponding wavespeed is written by

or

$$
z=C_{R} \pm \sqrt{\vartheta}
$$

$$
C=\bar{U}-C_{R} \pm \sqrt{\vartheta} \text {. }
$$

According to the computations above, though it is a rough estimation, we may temporarily conclude that the new types of instability are unlikely to occur in the presence of the divergent part of the wind component, especially for the longerand shorter-wave regions of the disturbance. 


\section{Motion of the non-geostrophic wind component}

In the previous section, the influence of the non-geostrophic wind component upon the stability properties of the baroclinic atmosphere was examined, and it was tentatively concluded that the non-geostrophic wind component does not cause any new type of baroclinic instability.

In the following discussions, we will neglect the terms relating to the mean zonal current, and investigate only the motion of the perturbations added.

The fundamental equations taken up are the same as used in the previous section, except for the absence of the terms containing the mean current. Therefore, they take the following forms for the simple two-level model of the baroclinic atmosphere:

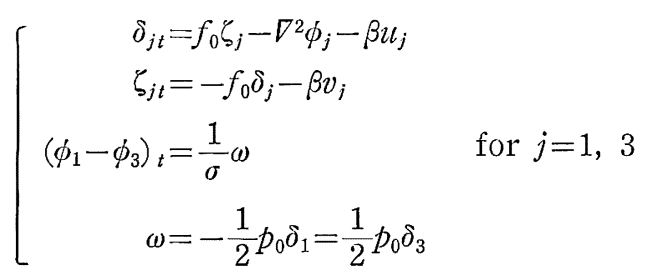

For the sake of brevity, we will assume in this section that the disturbance quantities are independent of the meridional direction, so we can put $\partial / \partial y=0$.

The relations between vorticity, isobaric height and vertical motions are obtained in the following way, by the introduction of the differential operator $D$ :

$$
\begin{aligned}
D & \equiv \beta+\frac{\partial^{2}}{\partial x \partial t} \\
D\left(v_{1}-v_{3}\right) & =\frac{4 f_{0}}{p_{0}} \omega \\
D\left(v_{1}-v_{3}\right) & =\frac{4 f_{0}}{p_{0}} \sigma\left(\phi_{1}-\phi_{3}\right)_{\iota} \\
\left(\phi_{1}-\phi_{3}\right)_{t} & =\frac{1}{\sigma} \omega .
\end{aligned}
$$

Combining the above three formulae into an equation of the single dependent variable $q$, which represents $\left(v_{1}-v_{3}\right),\left(\phi_{1}-\phi_{3}\right)$ or $\omega$, we have

$$
\left(D^{2}+f_{0}{ }^{2} \frac{\partial^{2}}{\partial x^{2}}\right) \frac{\partial q}{\partial t}-\mu^{2} D\left(\frac{\partial^{3} q}{\partial x^{3}}\right)=0,
$$

where

$$
\mu^{2} \equiv \frac{1}{8} p_{0}^{2} \cdot\left[\phi_{p}(\ln \theta)_{p}\right]>0 .
$$

As a solution of the above equation, we assume the wave type solution propagating in $x$-direction without changing the amplitude, i.e.

$$
q \simeq e^{i k(x-c t)}, \quad k=2 \pi / L
$$


The frequency equation of the above formula comes out immediately by substitution of $q$ into the equation (15),

$$
\Delta(C)=C\left(C+C_{R}\right)^{2}-C_{I}^{2} C-\mu^{2}\left(C+C_{R}\right)=0,
$$

This is a cubic equation concerning the propagation speed $C$ which is to be solved.

For the small scale phenomena, in which the effect of the Rossby factor $\beta$ is negligible, the frequency equation involves the following three roots:

$$
\Delta(C)=0, \quad C=0, \quad C^{2}=\mu^{2}+C_{I}^{2}
$$

In this case, the fundamental equation reduces to the so-called telegraphic equation. The solution of this equation has been obtained by many authors, such as CAHN (1945), BoLIN (1953), and recently AsAI (1960) by numerical computation, associated with the problem of adjustment between the velocity and the pressure fields of the oceanic current or the atmospheric circulation.

$$
0=\frac{\partial^{3}}{\partial x^{2} \partial t}\left[\left(\frac{\partial^{2}}{\partial t^{2}}+f_{0}^{2}\right) \cdot q-\mu^{2} \frac{\partial^{2} q}{\partial x^{2}}\right] .
$$

The analytic solution of this equation is expressed by the so-called HeavisidePoincare solution under arbitrarily given initial conditions.

$$
\left\{\begin{aligned}
q(x \cdot 0) & =F(x) \\
\frac{\partial}{\partial t} q(x \cdot 0) & =G(x) .
\end{aligned}\right.
$$

Then

$$
\begin{aligned}
q(x \cdot t)= & \frac{1}{2 \mu} \int_{-\mu t}^{+\mu t} G\left(x+x^{\prime}\right) J_{0}\left(f_{0} \sqrt{t^{2}-\left(\frac{x^{\prime}}{\mu}\right)^{2}}\right) d x^{\prime} \\
& +\frac{1}{2 \mu} \int_{-\mu t}^{+\mu t} F\left(x+x^{\prime}\right) \frac{\partial}{\partial t} \cdot J_{0}\left(f_{0} \sqrt{t^{2}-\left(\frac{x^{\prime}}{\mu}\right)^{2}}\right) d x^{\prime}
\end{aligned}
$$

where $J_{0}$ means the Bessel function of the zero order.

As seen from the expression (17), the wave speed $C$ is expressed by the internal inertia-gravity wave and propagates in the positive and negative direction of the $x$ axis. The deformation of the wave form with respect to time can be followed by the above solution (18), or the approximate evaluation of the integral values due to the method of the steepest descent.

The influence functions of one-day after is plotted in Fig. 2. The main part of the disturbance amplitude seems to propagate with the characteristic velocity of the almost internal gravity wave, $\mu$. As seen from the figure, the qualitative character of the curve is well expressed by the approximate evaluation of the steepest descent method.

On the other hand, another solution of the equation, corresponding to $C=0$, offers the one which expresses the gradual approach to the geostrophic relation between the velocity and pressure fields. Here, we define the linearized potential vorticity $Q$ as follows : 

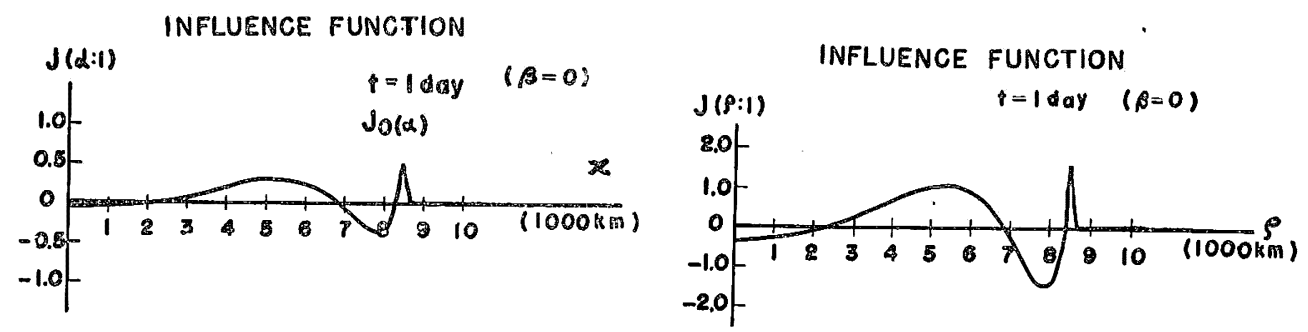

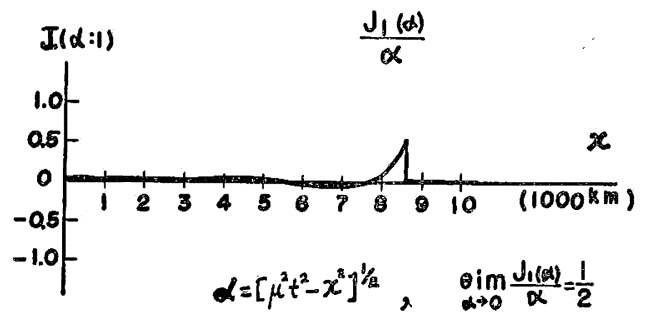

(a)

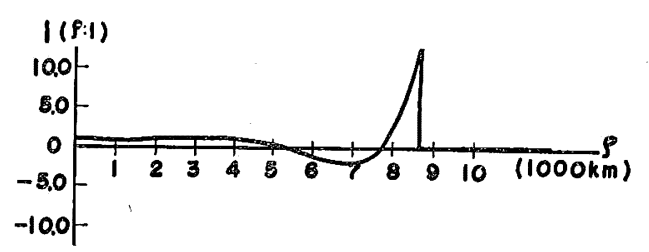

(b)

Fig. 2. Influence function, only right part is shown. $t=1$ day, $\mu=100 \mathrm{~m} / \mathrm{sec}$.

$$
Q(x) \equiv\left(v_{x}-\frac{4 f_{0}}{p_{0}} \sigma \phi\right),
$$

with

$$
v \equiv\left(v_{1}-v_{3}\right), \quad \phi \equiv\left(\phi_{1}-\phi_{3}\right) .
$$

The vorticity and adiabatic equation shows the conservation law of potential vorticity, that is to say,

where

$$
Q_{0}(x)=\left(v_{x}-\frac{4 f_{0}}{p_{0}} \sigma \phi\right)_{t=0}=\frac{1}{f_{0}}\left(\phi_{x x}-\tau^{2} \phi\right)_{t \rightarrow \infty}
$$

$$
\tau^{2} \equiv \frac{4 f_{0}^{2}}{p_{0}} \sigma=\frac{f_{0}^{2}}{\mu^{2}},
$$

expresses the critical wave number defined by EADY. The solution (18) shows the noise component propagating with the internal inertio-gravity wave, while the component satisfying the conservation law of the potential vorticity means the geostrophically balanced part.

The amplitude ratio between them can be estimated in the following way. If we assume the form of the monochromatic wave $e^{i k x}$ in vorticity $v_{x}$ or equivalently velocity $v$ and geopotential $\phi$-fields, then the conservation law of the potential vorticity enables us to evaluate the amplitude ratio at the initial and final states of the motion.

$$
v_{0}, \phi_{0} \simeq e^{i k x} .
$$

Then, $Q(x)$ offers the following relations:

$$
v_{s}=\frac{k^{2}}{k^{2}+\tau^{2}} v_{0}, \quad \phi_{s}=\frac{\tau^{2}}{k^{2}+\tau^{2}} \phi_{0}
$$


As easily seen from the expressions, the amplitude ratio $\left(v_{s} / v_{0}\right)$, or $\left(\phi_{s} / \phi_{0}\right)$, is a function of the wave number $k$, and the portion of the stationary part in the velocity field, $v_{s}$ to $v_{0}$, becomes smaller as the wavelength $L$ of the disturbance increases, while the relation of $\phi_{s}$ to $\phi_{0}$ becomes the reverse to the case of $v$.

In Fig. 3, the disturbance wavelength $L$ is taken as an abscissa and in the ordinate the amplitude ratios of velocity and isobaric height are taken, respectively. For the velocity field, the ratio of the meteorologically significant part to the noise part becomes smaller as the wavelength increases. The solid and the dotted line in the figure show the case of $\beta=0$, and $\beta \neq 0$, respectively. However, as easily recognized from the figure, there seems to be little difference between them, while the situation is quite opposite in the pressure field, that is, the ratio of the significant part to the noise part becomes larger as the increase of the wavelength. The amplitude ratio be-
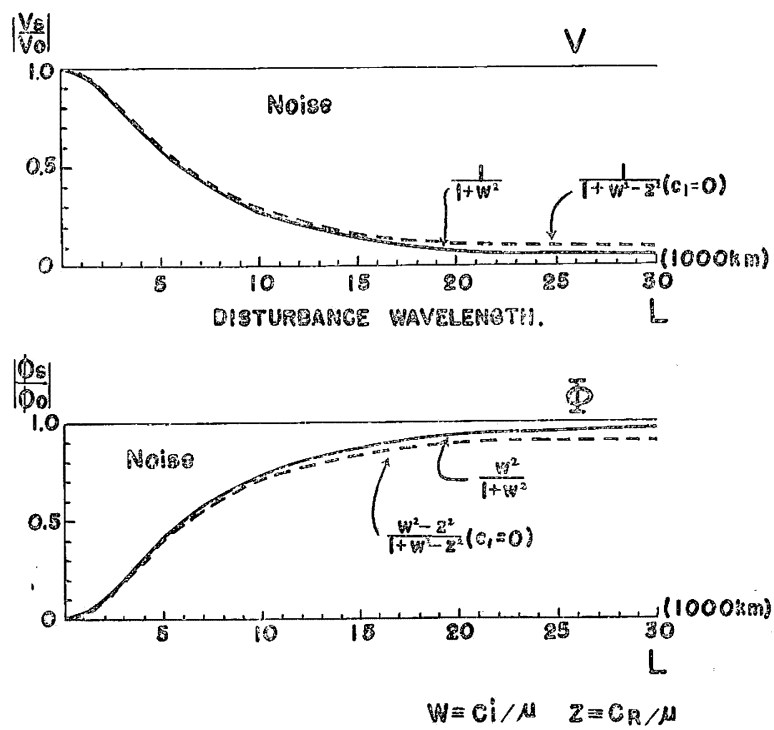

Fig. 3. The ratio of noise part to meteorologically significant part. The ordinate is expressed by $v_{s} / v_{0}, \phi_{s} / \phi_{0}$, the disturbance wavelength $L$, the abscissa. comes $1 / 2$ for the disturbance of the wavelength $6,000 \mathrm{~km}$, provided that the speed of the internal gravity wave is $100 \mathrm{~m} / \mathrm{sec}$, and the value of Coriolis parameter is taken at $45^{\circ} \mathrm{N}$.

On the other hand, for arbitrarily given values of $v$ and $\phi$, we can estimate the geopotential height $\phi_{s}(x)$ at an equilibrium state under the boundary conditions of $\phi_{s} \rightarrow 0$, as $|x| \rightarrow \infty$, in the following way:

$$
\phi_{s}(x)=\int_{-\infty}^{+\infty} G\left(x: x^{\prime}\right) Q_{0}\left(x^{\prime}\right) d x^{\prime},
$$

where $Q_{0}$ means the linearized potential vorticity at the initial moment, and the Green function is defined by

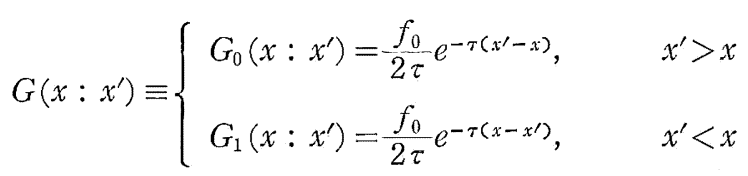

For large-scale phenomena, in which the Rossby factor $\beta$ is not negligible, we need to solve the frequency equation in its complete form

$$
\begin{aligned}
\Delta(C) & =C^{3}+2 C_{R} C^{2}+\left(C_{R}^{2}-C_{I}^{2}-\mu^{2}\right) C-\mu^{2} C_{R} \\
& =C\left(C+C_{R}+\sqrt{\mu^{2}+C_{I}^{2}}\right)\left(C+C_{R}-\sqrt{\mu^{2}+C_{I}^{2}}\right)-\mu^{2} C_{R}=0 .
\end{aligned}
$$




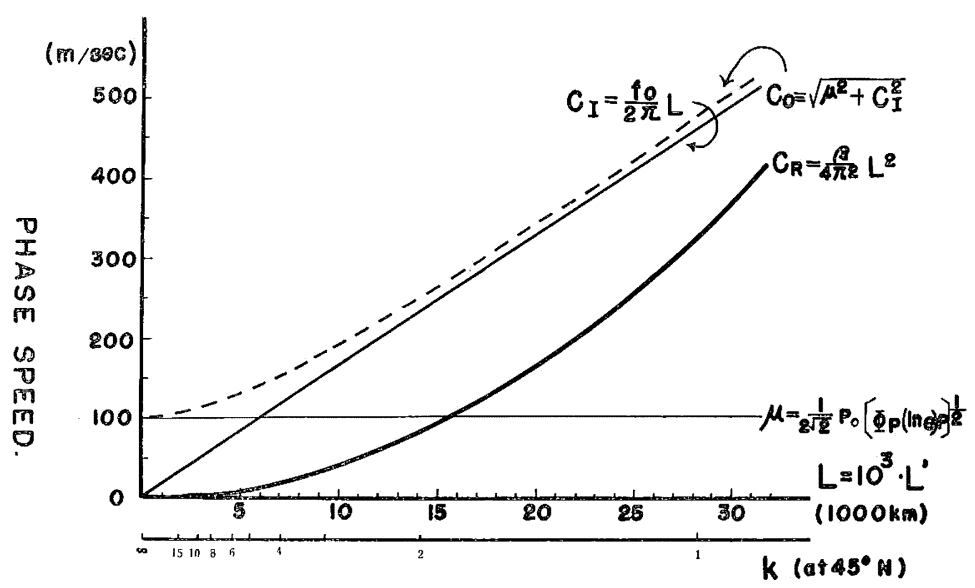

Fig. 4. Dependence of wave velocity upon the disturbance wavelength $L$. $\mu$, the interval gravity wave, $C_{I}$, the inertial wave, and $C_{R}$, the Rossby wave at $45^{\circ} \mathrm{N}$.

Fig. 4 shows the changes of the phase speed $C$ of the internal gravity wave, Rossby wave and inertia wave with respect to the disturbance wavelength $L$ at $45^{\circ} \mathrm{N}$. As easily seen from the figure, the speed of the inertia and internal gravity waves is rather large compared with the Rossby wave speed for the ranges of the wave number larger than 3. For a sufficiently long wavelength, however, the Rossby wave and the inertia wave become predominant. The dependence of the elementary waves $\mu, C_{I}$ and $C_{R}$ upon the disturbance wavelength $L$ suggests that, in the longer wave range, the rightmost term, $\mu^{2} C_{R}$, of the equation $(15)^{\prime}$ may be smaller in magnitude than the remaining terms on the right hand side. Therefore, we can obtain the following three roots as a first approximation:

$$
\left\{\begin{array}{l}
C_{1} \simeq 0 \\
C_{2} \simeq-C_{R}+\sqrt{\mu^{2}+C_{I}^{2}} \\
C_{3} \simeq-C_{R}-\sqrt{\mu^{2}+C_{I}{ }^{2}},
\end{array}\right.
$$

$C_{2}$ and $C_{3}$ are composed of the simple algebraic addition of the Rossby wave and the inertial-internal gravity wave. The second approximation of $C_{1}$ gives the following form, which means the wave slowly moving westward and corresponds to a meteorologically significant part of the motion.

$$
C_{1} \simeq \frac{-\mu^{2}}{\mu^{2}+C_{I}^{2}-C_{R}^{2}} \cdot C_{R}
$$

This is obtained by equating the third and fourth terms on the right hand side of the equation $(15)^{\prime}$. In Fig. 5, the phase velocities are plotted, and the solid line represents the result of the numerical solution of the frequency equation, and the dotted line means the one computed from the approximate expressions (19) and $(19)^{\prime}$ above. The coincidence between the solid and dotted lines is remarkably good, and it should be remarked that, in the longer wave range, the propagation velocity of waves $C_{2}$ and $C_{3}$ is considerably large. Therefore, when we consider 
the forecasting use of the primitive form of the equation, and furthermore the amount of energies inherited by the non-geostrophic wind component and if it is large, then the modification of the forecast equation itself or the suitable treatment of the initial data must be necessary, because the noise component acts not only as a "noise" obscuring the meteorologically significant motion, but also from the point of view of the computational stability problem, the large number of time step must be required in order to safely follow the evolution of the atmospheric flow system.

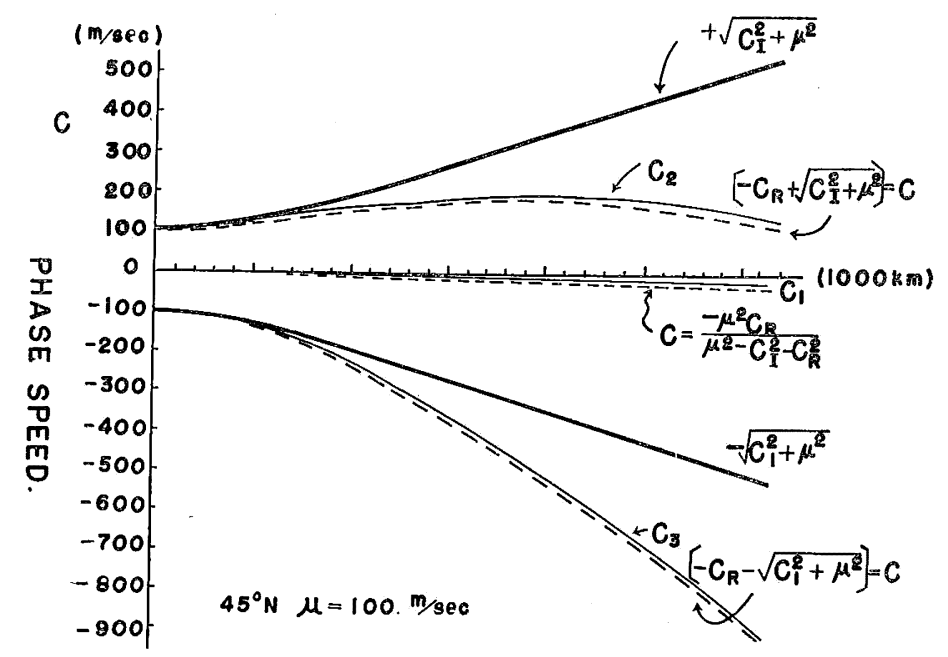

Fig. 5. Distribution of $C_{1}$, the meteorologically significant wave and $C_{2}, C_{3}$ the noise part of the wind.

The solid line shows the numerical solution and the dotted line, the analytic solution of the frequency equation.

Correspondingly, the initial treatment of the raw data becomes an important problem in to using the system of primitive equations. First, Hinkelmann (1951) considered the subject in the case of the barotropic divergent atmosphere, and recently, Sasaki (1958), Phillips (1959) and Matsumoto (1961) investigated the problem in the simple baroclinic atmosphere.

In the next section, we will examine the kinetic energy partitions between the divergent and the rotational part of the wind by using the data of the actual atmosphere.

\section{Energetical consideration}

The equations of motion without involving the terms of the mean zonal current and $\beta$-effect are given by

$$
\left\{\begin{array}{l}
\zeta_{t}=-f_{0} \nabla^{2} x \\
\delta_{t}=f_{0} \nabla^{2} \psi-\nabla^{2} \phi .
\end{array}\right.
$$

Here, we will assume that the initial fields of the divergence, vorticity and geo- 
potential height satisfy the following relations:

$$
\left\{\begin{aligned}
\zeta=\nabla^{2} \phi & =-m^{2} \phi \\
\nabla^{2} \phi & =-m_{0}^{2} \phi \\
\delta=\nabla^{2} x & =-\lambda^{2} x,
\end{aligned}\right.
$$

where $m^{2}, m_{0}{ }^{2}$, and $\lambda^{2}$ mean the characteristic wavenumber of the corresponding fields. Therefore, we may easily calculate the time tendency of the rotational or divergent part of the kinetic energy as follows:

$$
\left\{\begin{array}{l}
K_{\varphi t}=\int(\zeta \psi)_{t} \cdot d \tau=f_{0}\left(\lambda^{2}+m^{2}\right) \int \phi x d \tau \\
K_{x t}=\int(\delta x)_{t} \cdot d \tau=\left(\lambda^{2}+m_{0}^{2}\right) \int \phi x d \tau-f_{0}\left(\lambda^{2}+m^{2}\right) \int \dot{\psi} x d \tau .
\end{array}\right.
$$

Then, we define the ratio $R$ of the energies in the following way as a function of the characteristic wavenumber:

$$
R \equiv\left[\frac{K_{\varphi t}}{K_{x t}}\right]^{-1}=-1+\frac{\lambda^{2}+m_{0}^{2}}{\lambda^{2}+m^{2}} \frac{\int x \phi d \tau}{f_{0} \int x \phi d \tau} .
$$

In order to investigate in more detail, we expand $\phi, \phi$, and $x$-field into onedimensional Fourier series along the latitudinal direction,

$$
\left[\begin{array}{l}
\chi(x)=\sum_{k} X(k) e^{i k x} \\
\phi(x)=\sum_{k} \Phi(k) e^{i k x} \\
\phi(x)=\sum_{k} \Psi(k) e^{i k x}
\end{array}\right.
$$

where $k$ means the wavenumber. By substitution of the above quantities into the right hand side of the expression $R$, we have

$$
R=-1+\frac{1}{f_{0}} \frac{\sum_{k} k^{2} \Phi(k) \tilde{X}(k)}{\sum_{k} k^{2} \Psi(k) \tilde{X}(k)},
$$

where $\tilde{X}$ means the complex conjugate quantity of $X$. For each wave component, it takes a simple form,

$$
R(k)=-1+\frac{\Phi(k)}{f_{0} \Psi(\bar{k})}
$$

By using the data carefully analysed by Murakami, Tomatsu et al., the above quantity is estimated. The data are analysed for limited regions of the northern hemisphere extending from $160^{\circ} \mathrm{E}$ to $20^{\circ} \mathrm{W}$ along the longitude and from $15^{\circ}$ to $85^{\circ} \mathrm{N}$ in latitudinal direction for each standard levels. Among them, the data of 300,500 and $700 \mathrm{mb}$ levels were selected, and the meteorological quantities $U, V$, 
$\omega$ and $\phi$, in which the effect of orography is taken into account, along $45^{\circ} \mathrm{N}$ latitude were developed into Fourier series up to wavenumber 9.

Fig. 6 shows the distribution of the normalized spectrum function of the isobaric height $\phi$, vorticity $\zeta \equiv \partial v / \partial x$ and the vertical motion for 300,500 and $700 \mathrm{mb}$ level surfaces. From the figure, the predomination of the component of wavenumber 2 is seen for $\phi$-spectrum at $500,700 \mathrm{mb}$ levels.

\section{SPECTRUM DISTRIBUTION}
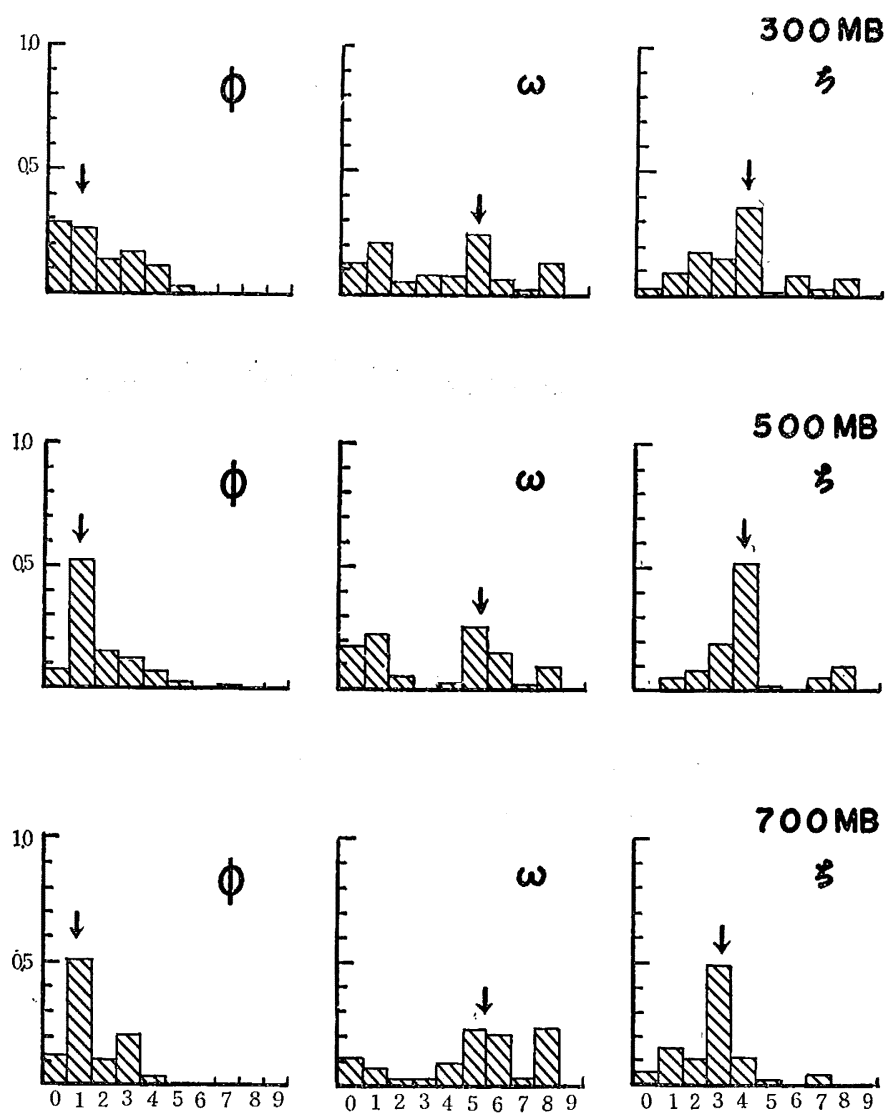

Fig. 6. Normalized spectrum function of the isobaric height $\phi$, the vertical velocity $\omega$, and the vorticity $\zeta$, respectively.

Concerning $\omega$-spectrum, on the other hand, no remarkable component is recognized, but the amplitudes of the component of wavenumber 2, 6 and 9 are comparatively larger than others for each of the three layers. As to vorticity spectrum $\zeta$, the component of wavenumber 5 for $300,500 \mathrm{mb}$ levels and of wavenumber 4 for $700 \mathrm{mb}$ level is outstanding.

On the other hand, we can easily estimate the ratio of $\Phi(k) / f_{0} \Psi(k)$ for each wavenumber $k$. In Fig. 7 , the ratio is exhibited. We can easily observe that the 
values are nearly equal to unity, that is to say, $R \simeq 0$, except for those of wavenumber 6 and 7 for 300,500 and $700 \mathrm{mb}$ levels.

Therefore, we may say that, for the components of wavenumber 6 and 7 , the time tendency of kinetic energy integral of the divergence component is larger than that of the rotational component. We may infer that the divergent part of the wind will be important at these wavenumber components.
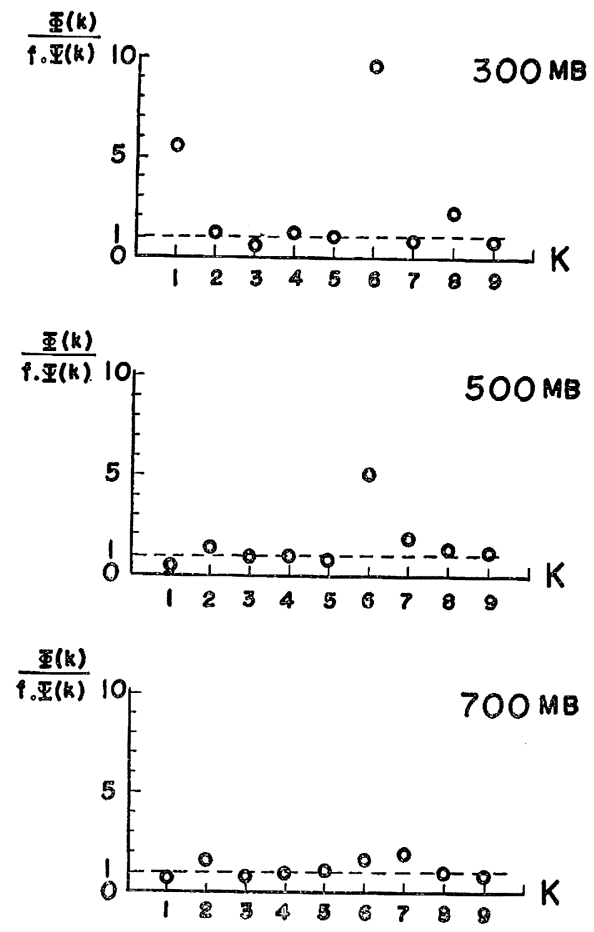

Fig. 7. Distribution of the ratio, $\Phi(k) / f_{0} \Psi(k)$ against wavenumber $k$.

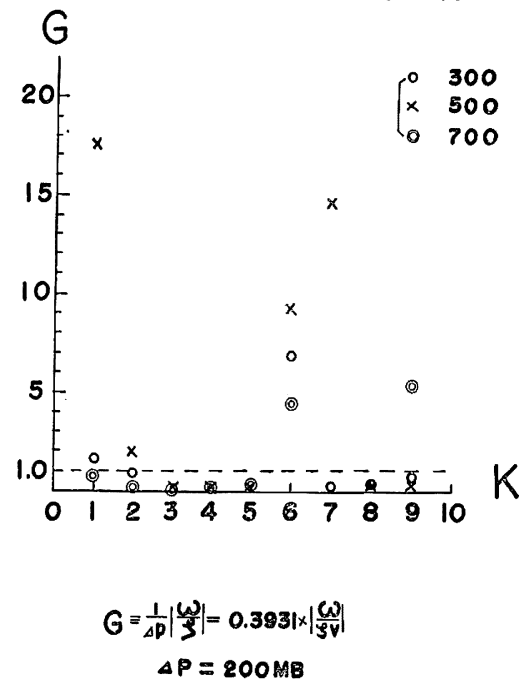

Fig. 8. $G$-number is plotted in the ordinate, and the disturbance wavenumber $k$, the ordinate.

This can be investigated in another way, namely, by examination of the ratio of the divergence and the rotational part of the wind itself. For this purpose, the non-dimensional quantity is defined by

$$
G \equiv \frac{1}{\Delta P}\left|\frac{\omega}{\zeta}\right|=\frac{1}{\Delta P}\left|\frac{\nabla^{2} \chi}{\nabla^{2} \psi}\right|
$$

The value of $\Delta p$ is taken to be $200 \mathrm{mb}$ and is taken to make $G$ a non-dimensional number. The value of $G$ is plotted in Fig. 8. It is immediately seen that values at wavenumber 6 and 7 are large compared with the other components. So it may be said that the divergence effect will play an important role in the large-scale component of wavenumber 1 and the intermediate component of wavenumber 6 and 7. The former is considered to be the divergence effect of the ultra long wave type, the significance of which was first stressed by Burger based on the 
scale-consideration of the atmospheric motion. The latter is due to the development of the baroclinic disturbance usually observed in the atmosphere.

Acknowledgements_- The present author would like to express his thanks to Dr. H. Arakawa for his encouragement, to Dr. S. Matsumoto for his kind discussions, and also to the members of the Forecast Research Laboratory of the Meteorological Research Institute.

\title{
References
}

Asat, T., 1960: to be published.

Bours, B., 1953: The adjustment of a nonbalanced velocity field towards geostrophic equilibrium in a stratified fluid. Tellus, 5, 3, 373-386.

BURger, A.P., 1958: Scale consideration of planetary motions of the atmosphere. Tellus, $10,2,195-206$.

$\mathrm{C}_{\mathrm{AHN}}, \mathrm{A} ., 1945$ : An investigation of the free oscillations of a simple current system. J. Met., 2, 113-119.

EADY, E.T., 1949: Long waves and cyclone waves. Tellus, 1, 33-52.

Eliassen, A., 1957: A procedure for numerical integration of the primitive equations of the two-parameter model of the atmosphere. UCLA, Scientific Report, No. 4.

Gамво, K., 1957: Three-dimensional treatment of equations of motion without using the geostrophic approximation. J.M.S.J., 35, 1-11.

Hinkelmann, K., 1951: Der Mechanismus des Meteorologischen Lärmes. Tellus, 3, 4, 285-296.

Матsuмото, S., 1961: A note on geostrophic adjustment and gravity wave in the atmosphere. J.M.S.J. 39, 18-28.

Philulps, N.A., 1959: On the problem of initial data for the primitive equations. Tellus, 12, 2, 121-126.

SASAKI, Y., 1958: An objective analysis based on the variational method. J.M.S.J. 36, 77-88. Tномpson, P.D., 1956: A theory of large-scale disturbances in non-geostrophic flow. J. Met., $13,3,251-261$.

\section{非地衡風を考慮した大規模な傾圧擾乱の運動について}

\author{
相原正彦 \\ オイラー形式の運動方程式, 断熱方程式を用いて, 大気中の大規模運動を調べた。方程式の中とは, 気 \\ 象学的に有意義な運動々, 所謂, “Noise” が含まれる。非地衡風战分の存在による地衡風㑯压擾乱の安定 \\ 度飞及ぼす影響及び，それらの伝播速度を調べた。采た，大気の $300 ， 500 ， 700 \mathrm{mb}$ の資料を用いて，風 \\ の発散及び回転成分のもつ運動ニネルギー比を求めた。
}

